\title{
Global Exponential Stability of Discrete-Time Neural Networks with Time-Varying Delays
}

\author{
S. Udpin ${ }^{1}$ and P. Niamsup ${ }^{2,3}$ \\ ${ }^{1}$ Department of Mathematics, Kasetsart University, Kamphaeng Saen Campus, Nakhonpathom 73140, Thailand \\ ${ }^{2}$ Department of Mathematics, Chiang Mai University, Chiang Mai 50200, Thailand \\ ${ }^{3}$ Center of Excellence in Mathematics CHE, Si Ayutthaya Road, Bangkok 10400, Thailand
}

Correspondence should be addressed to S. Udpin; faasstu@ku.ac.th

Received 13 August 2013; Revised 20 November 2013; Accepted 21 November 2013

Academic Editor: Lifeng Ma

Copyright (C) 2013 S. Udpin and P. Niamsup. This is an open access article distributed under the Creative Commons Attribution License, which permits unrestricted use, distribution, and reproduction in any medium, provided the original work is properly cited.

This paper presents some global stability criteria of discrete-time neural networks with time-varying delays. Based on a discrete-type inequality, a new global stability condition for nonlinear difference equation is derived. We consider nonlinear discrete systems with time-varying delays and independence of delay time. Numerical examples are given to illustrate the effectiveness of our theoretical results.

\section{Introduction}

In recent years, neural networks (NNs) have been investigated extensively due to their broad applications in information processing problems, associative memory, parallel computation, pattern recognition, signal processing, and optimization problems. It is well known that delays are often the sources of instability and oscillation in system. In practical studies, discrete-time systems have been used for a variety of phenomena in electrical networks, genetics, ecological systems, and so forth. Therefore, the stability analysis of discretetime neural networks (DNNs) with delays has become an important topic of theoretical studies in neural networks; for example, asymptotic stability and exponential stability of neural networks have been studied by many researchers. In [1], the authors have studied robust stability of discretetime linear-parameter-dependent (LPD) neural networks with time-varying delay. In order to derive stability criteria of discrete-time, one common approach is the use of appropriate inequalities for difference equations. Another approach is the use of Lyapunov stability theory. In [2], the authors have studied global exponential stability of impulsive discretetime neural networks with time-varying delays, based on some inequality analysis techniques. In [3], the authors have studied new discrete-type inequalities and global stability of nonlinear difference equation. In [4], the authors have studied global exponential stability of discrete-time Hopfield neural networks with variable by using the difference inequality. In [5], the authors have considered the problem of robust stability analysis of generalized neural networks with multiple discrete delays and multiple distributed delays by using the Lyapunov-Krasovskii functional method and the linear matrix inequality technique. In [6], the authors have studied delay-dependent exponential stability criteria for discretetime nonlinear system with multiple time-varying delays. In this paper, we propose to study global exponential stability of discrete-time neural networks with time-varying delays. In Section 2, we have introduced discrete-time neural networks with time-varying delays and presented some preliminaries. In Section 3, we have derived new discrete-type inequalities; global exponential stability criteria are derived by using new discrete-type inequalities. Finally, numerical examples are given to illustrate the effectiveness of our theoretical results.

\section{Notations, Definitions, and Preliminaries Results}

In this section, we give some notations definitions and preliminaries results which will be used throughout this paper. 
Let $\mathbb{R}$ denote the set of all real numbers; $\mathbb{R}^{+}$the set of positive real numbers; $\mathbb{R}_{0}^{+}$the set of nonnegative real numbers; $\mathbb{Z}$ the set of integers; and $\mathbb{Z}^{+}$the set of positive integers; $\mathbb{Z}^{-r}=\{z \in \mathbb{Z}: z \geq-r\}$. We consider the following discrete-time neural networks with time-varying delays:

$$
\begin{aligned}
u(n+1)= & C u(n)+A f(u(n)) \\
& +\sum_{i=0}^{r} B_{i} f\left(u\left(n-\tau_{i}(n)\right)\right)+b,
\end{aligned}
$$

where $u(n)=\left[u_{1}(n), \ldots, u_{p}(n)\right]^{T} \in \mathbb{R}^{p}$ is the neuron state vector, $b=\left[b_{1}, \ldots, b_{p}\right]^{T}$ is constant input vector, $\tau_{i}(n), i=$ $0,2, \ldots, r$ are positive integers denoting the time-varying delays satisfying

$$
0 \leq \tau_{i}(n) \leq \tau_{i}, \quad i=0,1,2, \ldots, r,
$$

where $\tau_{i} \geq 0$ are known positive integers, $C=\operatorname{diag}\left(c_{m}\right),\left(c_{m} \in\right.$ $(0,1)), A$ and $B_{i}$ are the interconnection matrices, and $f(x(\cdot))=\left[f_{1}\left(x_{1}(\cdot)\right), f_{2}\left(x_{2}(\cdot)\right), \ldots, f_{n}\left(x_{n}(\cdot)\right)\right]^{T}$ is the activation function where $f_{m}(\cdot), m=1, \ldots, n$ satisfy

$$
\left|f_{m}(x)-f_{m}(y)\right| \leq L_{m}|x-y|, \quad \forall x, y \in \mathbb{R} .
$$

The initial conditions of system (1) are of the form

$$
u_{m}(s)=\phi_{m}(s), \quad m=1,2, \ldots, n,
$$

where $\phi(s)=\left[\phi_{1}(s), \phi_{2}(s), \ldots, \phi_{n}(s)\right]^{T}$ is an initial functions, $s \in\left\{-\tau_{r},-\tau_{r}+1, \ldots, 0\right\}$, and $\tau_{r}$ is the maximum value of $\tau_{i}$. Let $u^{*}=\left[u_{1}^{*}, u_{2}^{*}, \ldots, u_{n}^{*}\right]^{T}$ be an equilibrium point of system (1). We shift the equilibrium point $u^{*}$ to the origin by using the transformation $x(\cdot)=u(\cdot)-u^{*}$. Then, we obtain the new system

$$
x(n+1)=C x(n)+A g(x(n))+\sum_{i=0}^{r} B_{i} g\left(x\left(n-\tau_{i}(n)\right)\right),
$$

where $x(n)=\left\{x_{1}(n), x_{2}(n), \ldots, x_{p}(n)\right\}$ is the state vector of the transformed system, $g(x(\cdot))=\left[g_{1}\left(x_{1}(\cdot)\right), \ldots, g_{n}\left(x_{n}(\cdot)\right)\right]^{T}$, where $g_{m}\left(x_{m}(\cdot)\right)=f_{m}\left(x_{m}(\cdot)+u_{m}^{*}\right)-f_{m}\left(u_{m}^{*}\right), m=$ $1,2, \ldots, n$ and the transformed activation functions satisfy the condition

$$
\left|g_{m}(x)\right| \leq L_{m}|x| .
$$

Definition 1 (see [4]). The equilibrium point at the origin of system (5) is globally exponentially stable if for any solution $x(n, \phi)$ with the initial condition $\phi$, there exist constant $\epsilon \in(0,1)$ and $D>0$ such that

$$
\|x(n)\| \leq D\|\phi\| \epsilon^{n} \quad \forall n \leq 0,
$$

where $\|\phi\|=\max _{s \in\left[-\tau_{r}, 0\right]}\{\|\phi(s)\|\}$.

\section{Main Result}

Throughout this section, we denote $x(n)$ by $x_{n}$. In this section, we provide global exponential stability criteria for system (5). First, we introduce new discrete-type inequalities which will be used to derive global exponential stability condition.

Lemma 2. Let $q_{i} \in \mathbb{R}_{0}^{+}, k_{i} \in \mathbb{Z}^{+}, i=1, \ldots r ; p, q_{r} \in \mathbb{R}^{+}$, where $0 \leq k_{0}<k_{1}<\cdots<k_{r}$ and $\sum_{i=0}^{r} q_{i}\left(k_{i}+1\right)<p \leq 1$, and let $\left\{x_{j}\right\}_{j \in \mathbb{Z}^{-k_{r}}}$ be a sequence of real numbers satisfying the inequality

$$
\Delta x_{n} \leq-p x_{n}+\sum_{i=0}^{r} q_{i}\left(k_{i}+1\right) \sum_{j=0}^{k_{i}} x_{n-j}, \quad n \in \mathbb{Z}^{0},
$$

where $\Delta x_{n}=x(n+1)-x(n)$. Then, there exists $\lambda_{0} \in(0,1)$ such that

$$
x_{n} \leq \max \left\{0, x_{0}, x_{-1}, \ldots, x_{-k_{r}}\right\} \lambda_{0}^{n}, \quad n \in \mathbb{Z}^{0} .
$$

Moreover, $\lambda_{0}$ might be chosen as the smallest positive root of the polynomial

$$
P(\lambda)=\lambda^{k_{r}+1}-(1-p) \lambda^{k_{r}}-\sum_{i=0}^{r} q_{i} \sum_{j=0}^{k_{i}} \lambda^{k_{r}-j}
$$

which lies in the interval $(0,1)$.

Proof. Let $\left\{y_{n}\right\}$ be a solution of the difference equation

$$
\Delta y_{n}=-p y_{n}+\sum_{i=0}^{r} q_{i}\left(k_{i}+1\right) \sum_{j=0}^{k_{i}} y_{n-j}, \quad n \in \mathbb{Z}^{0} .
$$

From $q_{i} \in \mathbb{R}_{0}^{+}$and $0<p<1$, one may follow the proof of Theorem 2.1 in [3] to show that if $x_{n}$ satisfies (8) and $x_{n} \leq$ $y_{n}$ for $n=-h_{r}, \ldots, 0$, then $x_{n} \leq y_{n}$ for all $n \in \mathbb{Z}^{0}$. For given $K>0$ and $\lambda \in(0,1)$, the sequence $\left\{y_{n}\right\}$ defined by $y_{n}=K \lambda^{n}$ is a solution of (11) if and only if $\lambda$ is a root of the polynomial (10). Since $\lim _{\lambda \rightarrow 0^{+}} P(\lambda)=-q_{r}<$ 0 and $P(1)=p-\sum_{i=0}^{r} q_{i}\left(k_{i}+1\right)>0$, it follows from continuity of $P$ that there exists a smallest real number $\lambda_{0} \in(0,1)$ such that $P\left(\lambda_{0}\right)=0$. Thus, for any $K \in \mathbb{K}_{0}^{+}$, the sequence $\left\{K \lambda_{0}^{n}\right\}$ is a solution of (11). Let $K_{0}=\max \left\{0, x_{n}, x_{-1}, \ldots, x_{-k_{r}}\right\}$. Therefore, $y_{n}=\left\{K_{0} \lambda_{0}^{n}\right\}$ is a solution of (11) and obviously, we have $x_{n} \leq y_{n}$, for $n=-k_{r}, \ldots, 0$. Hence, by using the first part of the proof, we conclude that $x_{n} \leq y_{n}=K_{0} \lambda_{0}^{n}, n \in$ $\mathbb{Z}^{0}$.

Now, we will provide a global exponential stability condition for system (5).

Theorem 3. The equilibrium point at the origin of system (5) is globally exponentially stable if

$$
c_{\max }+l\|A\|+l \sum_{i=0}^{r}\left(\tau_{i}+1\right)\left\|B_{i}\right\|<1,
$$

where $l=\max _{m}\left(L_{m}\right), c_{\max }=\max _{m}\left(c_{m}\right)$, and $\max _{m}\left(L_{m}\right)$ is maximum of vector. 
Proof. Let $z_{n}=\left\|x_{n}\right\|$. Then, from (5), the difference of system is given by

$$
\begin{aligned}
\Delta z(n)= & \left\|x_{n+1}\right\|-\left\|x_{n}\right\| \\
= & \left\|C x_{n}+A g\left(x_{n}\right)+\sum_{i=0}^{r} B_{i} g\left(x_{n-\tau_{i}(n)}\right)\right\|-\left\|x_{n}\right\| \\
\leq & \left\|C x_{n}\right\|+\left\|A g\left(x_{n}\right)\right\|+\sum_{i=0}^{r}\left\|B_{i} g\left(x_{n-\tau_{i}(n)}\right)\right\|-\left\|x_{n}\right\| \\
\leq & -\left(1-c_{\max }-l\|A\|\right)\left\|x_{n}\right\|+l \sum_{i=0}^{r}\left\|B_{i}\right\|\left\|x_{n-\tau_{i}(n)}\right\| \\
\leq & -\left(1-c_{\max }-l\|A\|\right)\left\|x_{n}\right\|+l \sum_{i=0}^{r} \sum_{j=0}^{\tau_{i}}\left\|B_{i}\right\|\left\|x_{n-j}\right\| \\
\leq & -\left(1-c_{\max }-l\|A\|\right)\left\|x_{n}\right\| \\
& +l \sum_{i=0}^{r}\left(\tau_{i}+1\right)\left\|B_{i}\right\| \sum_{j=0}^{\tau_{i}}\left\|x_{n-j}\right\| \\
= & -p z_{n}+\sum_{i=0}^{r} q_{i}\left(k_{i}+1\right) \sum_{j=0}^{\tau_{i}} z_{n-j},
\end{aligned}
$$

where

$$
p=1-c_{\max }-l\|A\|, \quad q_{i}=\left\|B_{i}\right\|, \quad k_{i}=\tau_{i} .
$$

From Lemma 2, it follows assumptions of theorem that there exist $\lambda_{0} \in(0,1)$ such that

$$
z_{n} \leq \max \left\{0, z_{0}, z_{-1}, \ldots, z_{-\tau_{r}}\right\} \lambda_{0}^{n}, \quad n \in \mathbb{Z}^{0}
$$

Thus, we obtain

$$
\begin{aligned}
z_{n} & =\left\|x_{n}\right\| \leq \max \left\{0, z_{0}, z_{-1}, \ldots, z_{-\tau_{r}}\right\} \lambda_{0}^{n} \\
& =\max \left\{0,\left\|x_{0}\right\|,\left\|x_{-1}\right\|, \ldots,\left\|x_{-\tau_{r}}\right\|\right\} \lambda_{0}^{n} \leq\|\phi\| \lambda_{0}^{n} .
\end{aligned}
$$

By Definition 1, we conclude that (5) is globally exponentially stable. The proof is complete.

Remark 4. In our main result, we derived global exponential stability criteria for discrete-time neural networks with multiple time-varying delays by using discrete-type inequality. In [4], the global exponential stability criteria of discretetime Hopfield neural networks are given. Nevertheless, the stability criteria in [4] cannot be applied to discrete-time system with multiple time-varying delays.

\section{Numerical Example}

Example 1. Consider the NNs (1) with $r=2$ where

$$
\begin{gathered}
C=\left[\begin{array}{cc}
0.1 & 0 \\
0 & -0.15
\end{array}\right], \quad A=\left[\begin{array}{cc}
0.1 & -0.15 \\
-0.2 & 0.1
\end{array}\right], \\
B_{0}=\left[\begin{array}{cc}
0.2 & -0.1 \\
-0.1 & 0.15
\end{array}\right], \quad B_{1}=\left[\begin{array}{cc}
-0.1 & -0.15 \\
0.1 & -0.1
\end{array}\right] \\
b=[-3,-1], \\
\tau_{0}(n)=1+\sin \left(\frac{n \pi}{2}\right), \quad \tau_{1}(n)=2+\sin \left(\frac{n \pi}{2}\right), \\
f_{1}(s)=\tanh (-0.4 s)+0.2 \sin s, \quad f_{2}(s)=\tanh (0.2 s) .
\end{gathered}
$$

Then, $\|A\|_{1}=0.3,\left\|B_{0}\right\|_{1}=0.3,\left\|B_{1}\right\|_{1}=0.25, \tau_{0}=1, \tau_{1}=$ 2, $c_{\max }=0.15, L_{1}=0.5, L_{2}=0.2$, and $l=0.5$.

The equilibrium point of system in Example 1 is

$$
u^{*}=\left[u_{1}^{*}, u_{2}^{*}\right]^{T}=[-3.3507,-0.7254]^{T} .
$$

From which it follows that

$$
\begin{aligned}
c_{\max } & +0.5\|A\|_{1}+0.5\left(\tau_{0}+1\right)\left\|B_{0}\right\|_{1} \\
& +0.5\left(\tau_{1}+1\right)\left\|B_{1}\right\|_{1}=0.975<1 .
\end{aligned}
$$

Therefore, from Theorem 3, it follows that the equilibrium point at the origin of system (1) is globally exponentially stable.

The trajectory of solutions of Example 1 with the initial state $\left[\phi_{1}(s), \phi_{2}(s)\right]^{T}=[-3,1]^{T}$ for $s \in[-1,0]$ is shown in Figure 1 .

Example 2. Consider the NNs (1) with $r=2$ where

$$
\begin{gathered}
C=\left[\begin{array}{cc}
0.2 & 0 \\
0 & 0.1
\end{array}\right], \quad A=\left[\begin{array}{cc}
-0.1 & 0.15 \\
0.1 & -0.15
\end{array}\right] \\
B_{0}=\left[\begin{array}{cc}
0.15 & -0.1 \\
-0.2 & 0.05
\end{array}\right], \quad B_{1}=\left[\begin{array}{cc}
-0.15 & 0.1 \\
0.1 & -0.15
\end{array}\right] \\
b=[2,3], \\
\tau_{0}(n)=1+\sin \left(\frac{n \pi}{2}\right), \quad \tau_{1}(n)=2+\sin \left(\frac{n \pi}{2}\right), \\
f_{1}(s)=\tanh (-0.4 s)+0.2 \sin (s), \quad f_{2}(s)=\tanh (0.2 s) .
\end{gathered}
$$

Then $\|A\|_{\infty}=0.3,\left\|B_{0}\right\|_{\infty}=0.25,\left\|B_{1}\right\|_{\infty}=0.25, \tau_{0}=1$, $\tau_{1}=2, c_{\max }=0.2$, and $l=0.5$. The equilibrium point of system in Example 2 is

$$
u^{*}=\left[u_{1}^{*}, u_{2}^{*}\right]^{T}=[2.2435,3.5554]^{T} .
$$

From which it follows that

$$
\begin{aligned}
c_{\max } & +0.5\|A\|_{\infty}+0.5\left(\tau_{0}+1\right)\left\|B_{0}\right\|_{\infty} \\
& +0.5\left(\tau_{1}+1\right)\left\|B_{1}\right\|_{\infty}=0.975<1 .
\end{aligned}
$$




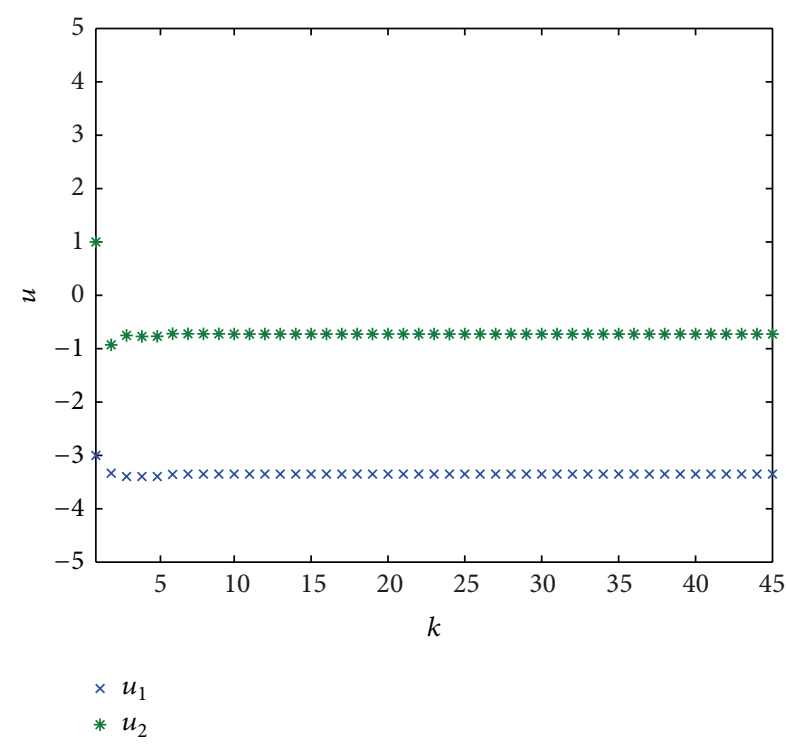

FIGURE 1: The trajectory of solutions of system (1) in Example 1.

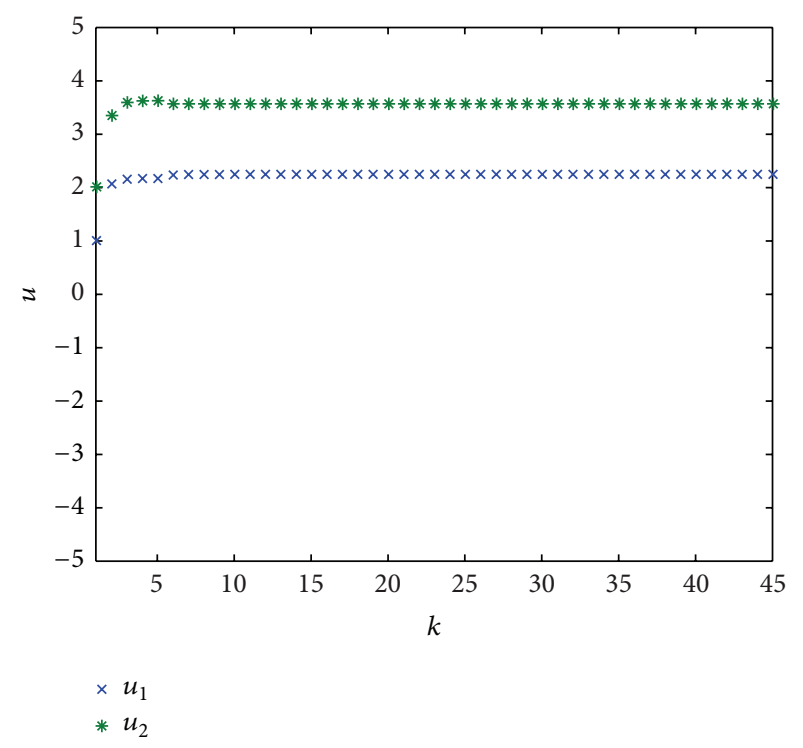

FIGURE 2: The trajectory of solutions of system (1) in Example 2.

Therefore, from Theorem 3, it follows that the equilibrium point at the origin of system (1) is globally exponentially stable.

The trajectory of solutions of Example 2 with the initial state $\left[\phi_{1}(s), \phi_{2}(s)\right]^{T}=[1,2]^{T}$ for $s \in[-1,0]$ is shown in Figure 2 .

\section{Conclusion}

In this paper, we obtained sufficient condition for global exponential stability of discrete-time neural networks with multiple time-varying delays. The stability criteria are derived by using a discrete-type inequality. Numerical examples are given to show the effectiveness of our theoretical results.

\section{Acknowledgments}

This research is supported by Research Promotion and Technology Transfer Center (RPTTC), Faculty of Liberal Arts and Science, Kasetsart University, Kamphaeng Saen Campus, Thailand, and the Center of Excellence in Mathematics, Thailand, and Commission for Higher Education, Thailand. The authors would like to thank anonymous reviewers for valuable comments and suggestions to improve the exposition of the paper.

\section{References}

[1] S. Udpin and P. Niamsup, "Robust stability of discrete-time LPD neural networks with time-varying delay," Communications in Nonlinear Science and Numerical Simulation, vol. 14, no. 11, pp. 3914-3924, 2009.

[2] H. Xu, Y. Chen, and K. L. Teo, "Global exponential stability of impulsive discrete-time neural networks with time-varying delays," Applied Mathematics and Computation, vol. 217, no. 2, pp. 537-544, 2010.

[3] S. Udpin and P. Niamsup, "New discrete type inequalities and global stability of nonlinear difference equations," Applied Mathematics Letters, vol. 22, no. 6, pp. 856-859, 2009.

[4] Q. Zhang, X. Wei, and J. Xu, "On global exponential stability of discrete-time Hopfield neural networks with variable delays," Discrete Dynamics in Nature and Society, vol. 2007, Article ID 67675, 9 pages, 2007.

[5] X. Liu and N. Jiang, "Robust stability analysis of generalized neural networks with multiple discrete delays and multiple distributed delays," Neurocomputing, vol. 72, no. 7-9, pp. 17891796, 2009.

[6] Y.-J. Sun and G.-J. Yu, "Delay-dependent exponential stability criteria for nonlinear time-varying discrete systems with multiple time delays," Journal of the Franklin Institute, vol. 334, no. 4, pp. 659-666, 1997. 


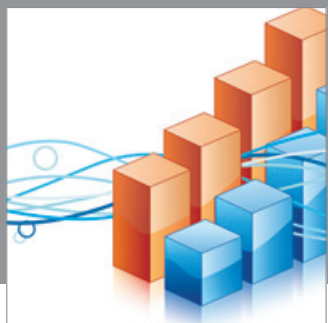

Advances in

Operations Research

mansans

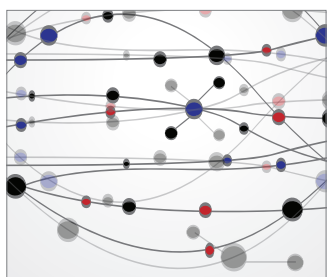

The Scientific World Journal
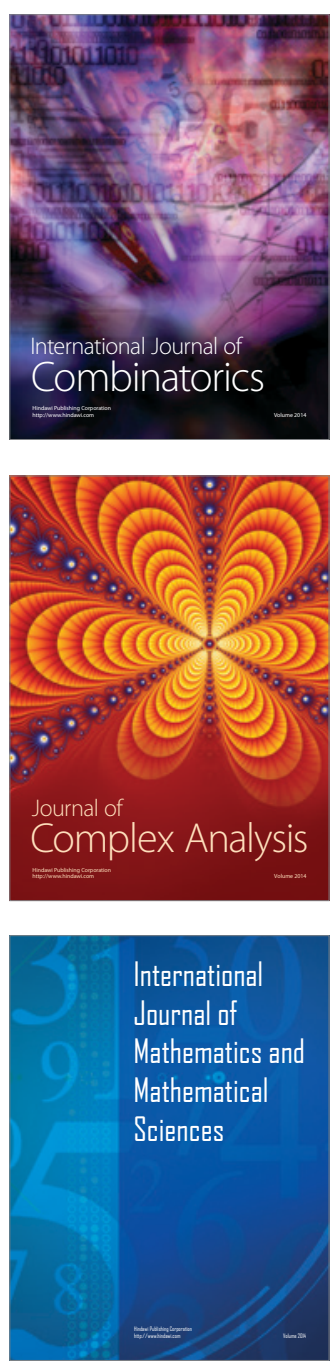
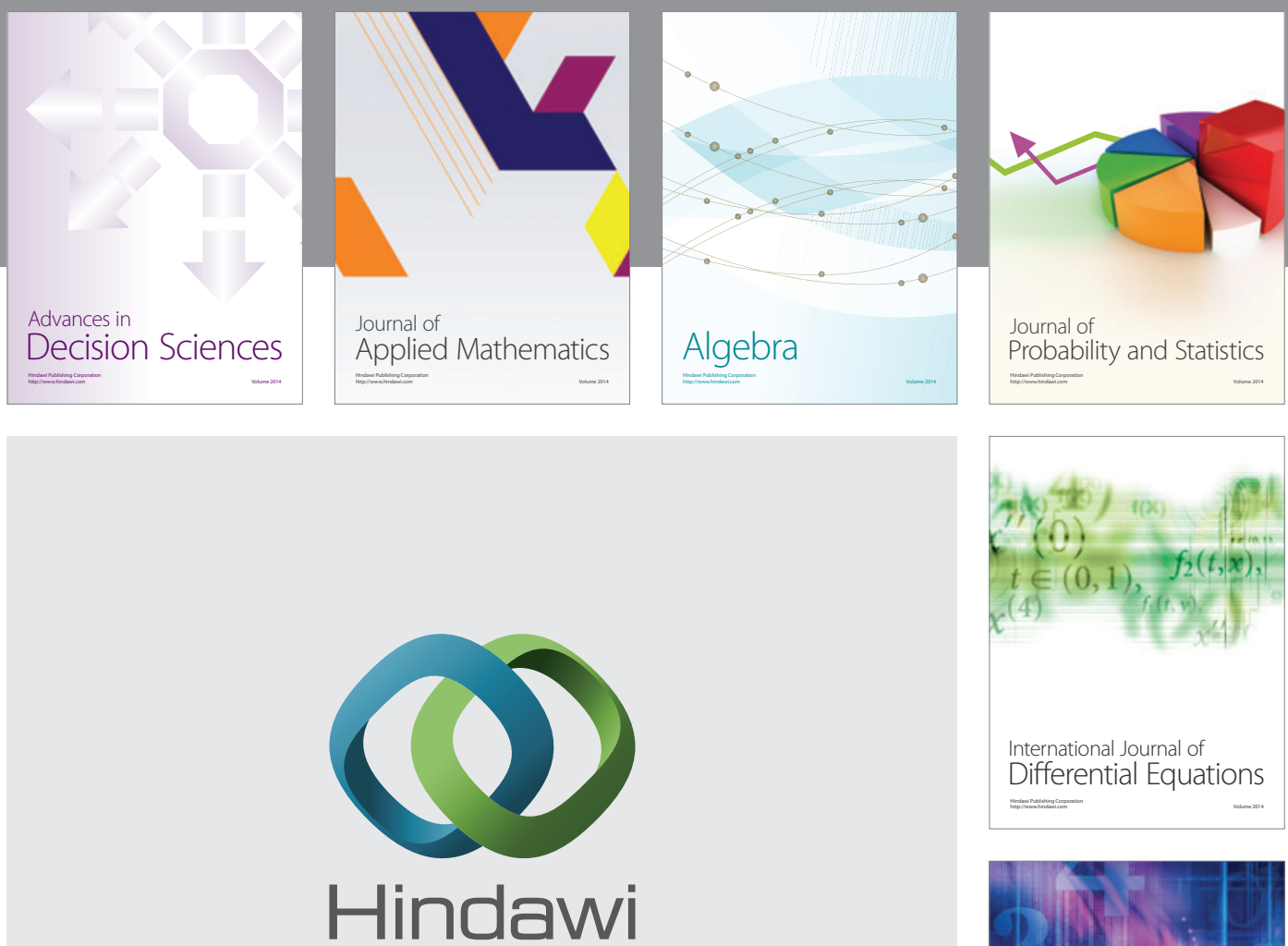

Submit your manuscripts at http://www.hindawi.com
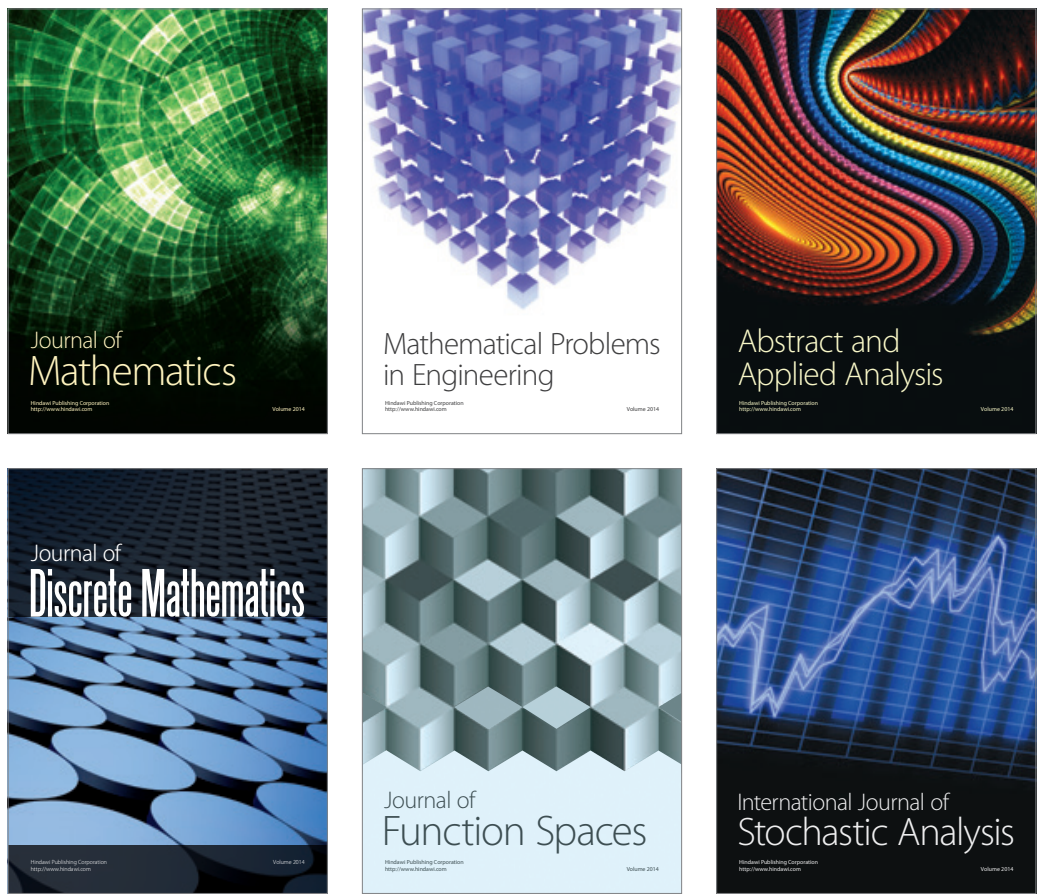

Journal of

Function Spaces

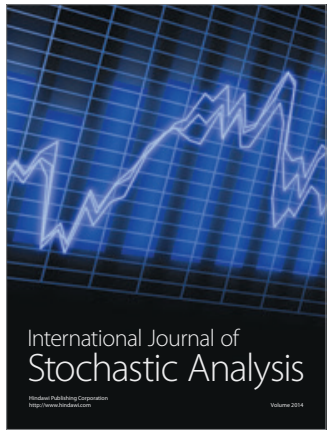

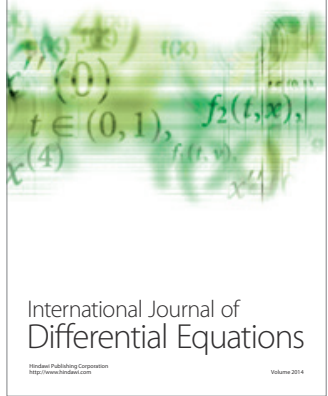
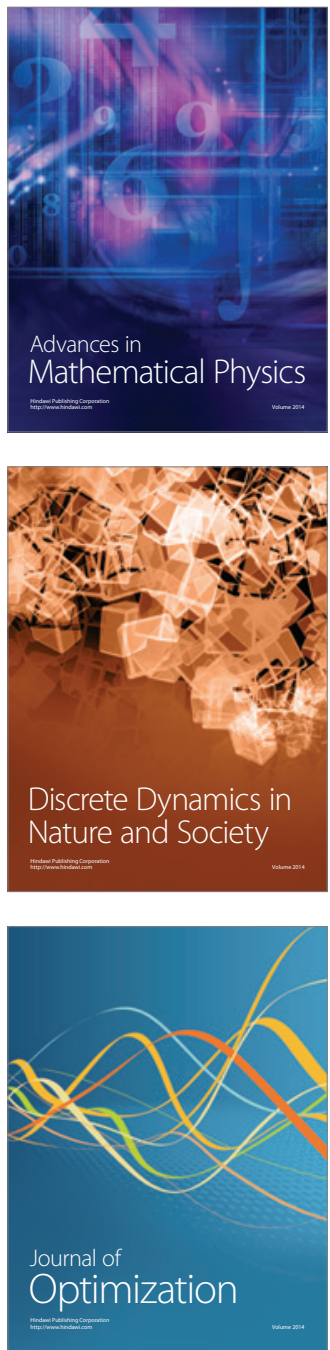